\title{
Design of the PIXIE Adiabatic Demagnetization Refrigerators
}

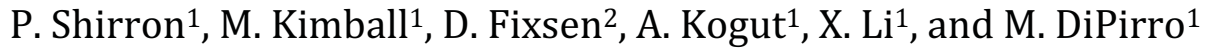 \\ ${ }^{1}$ NASA/Goddard Space Flight Center, Greenbelt MD 20771 USA \\ 2 University of Maryland, College Park MD 20740 USA
}

\begin{abstract}
The Primordial Inflation Explorer (PIXIE) is a proposed mission to densely map the polarization of the cosmic microwave background. It will operate in a scanning mode from a sun-synchronous orbit, using low temperature detectors (at $0.1 \mathrm{~K}$ ) and located inside a telescope that is cooled to approximately $2.73 \mathrm{~K}$ - to match the background temperature. A mechanical cryocooler operating at $4.5 \mathrm{~K}$ establishes a low base temperature from which two adiabatic demagnetization refrigerator (ADR) assemblies will cool the telescope and detectors. To achieve continuous scanning capability, the ADRs must operate continuously. Complicating the design are two factors: 1) the need to systematically vary the temperature of various telescope components in order to separate the small polarization signal variations from those that may arise from temperature drifts and changing gradients within the telescope, and 2) the orbital and monthly variations in lunar irradiance into the telescope barrels. These factors require the telescope ADR to reject quasi-continuous heat loads of 2-3 mW, while maintaining a peak heat reject rate of less than $12 \mathrm{~mW}$. The detector heat load at $0.1 \mathrm{~K}$ is comparatively small at $1-2 \mu \mathrm{W}$. This paper will describe the 3 stage and 2-stage continuous ADRs that will be used to meet the cooling power and temperature stability requirements of the PIXIE detectors and telescope.
\end{abstract}

\subsection{INTRODUCTION}

The Primordial Inflation Explorer (PIXIE)[1] in Figure 1 is a proposed mission to produce full-sky maps of the cosmic microwave background (CMB) polarization. The instrument will use a polarizing Michelson interferometer and optics cooled to the CMB temperature $(2.73 \mathrm{~K})$ to measure the difference spectrum between two orthogonal linear polarizations of light collected from two co-aligned telescope barrels. The weak polarization signals are read out with harp-string bolometers cooled to $0.1 \mathrm{~K}$. A calibrator can be moved to uncover one or both of the telescope barrels, so that the instrument can sample the difference between sky signals, or between sky signals and a calibrated $\sim 2.73 \mathrm{~K}$ black body.

The instrument achieves a high degree of systematic error rejection by its highlysymmetric, nulled design, and by nearly continuous calibration of the polarization signal. In addition, errors caused by to temperature gradients within telescope and optical components will be reduced by reduced by independently varying the temperature of these components by small $(5-20 \mathrm{mK})$ amounts relative to the $\mathrm{CMB}$, at unique frequencies. The impact is that the instrument's cooling system must not only lift heat conducted and 
radiated onto the instrument, but a significant amount of heat $(\sim 1 \mathrm{~mW})$ associated with these fluctuations.

PIXIE will be in a sun-synchronous, $575 \mathrm{~km}$ orbit, so that it can continuously map the sky to obtain full coverage every 6 months. As such, the instrument will experience some radiative input from the Moon during a portion of nearly every orbit. The input is relatively small when the Moon is full or new, but peaks when it is half full. The lunar irradiance into each open telescope barrel is a half-sinusoid with a maximum of $1 \mathrm{~mW}$. The orbit average is $0.5 \mathrm{~mW}$, and since this will occur each orbit for many days, the instrument cooling system must be designed as if this is a continuous heat load.

The PIXIE cryogenic system will use a set of radiation shields and a $4.5 \mathrm{~K}$ cryocooler to minimize heat flow into the instrument from the room temperature spacecraft and from the Sun and Earth. Two ADR assemblies will cool continuously from $4.5 \mathrm{~K}$ to $\sim 2.6 \mathrm{~K}$ for the telescope, and to $0.1 \mathrm{~K}$ for the detectors. This paper discusses the requirements, design and performance of the ADR system.

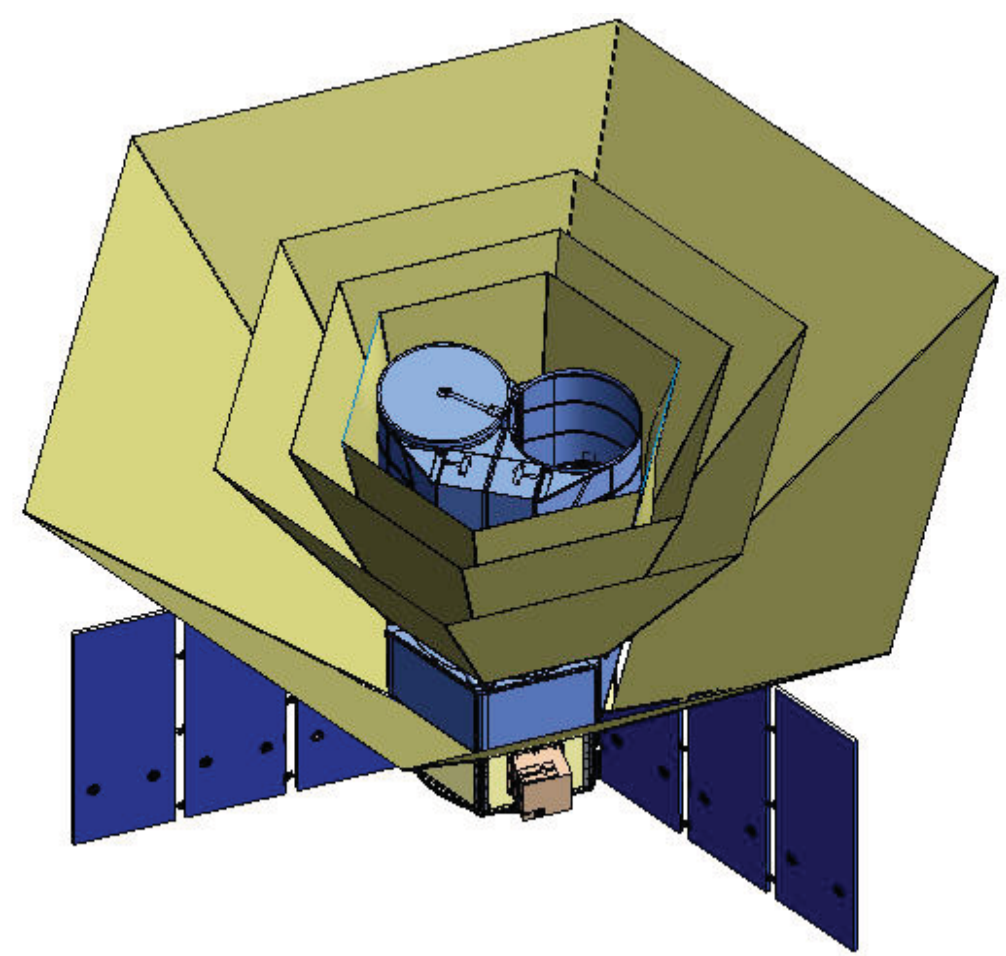

Figure 1. The PIXIE instrument (center) and 4 thermal shields. The outermost shield is approximately $3.5 \mathrm{~m}$ across when deployed. 


\subsection{ADR SYSTEM REQUIREMENTS}

Figure 2 is a heat map of the PIXIE instrument. The ADR is shown as a lumped unit, but consists of two separate assemblies: the iADR which cools the telescope and optical components, and the dADR which cools the detectors. A breakdown of heat loads for each is given in Table 1 . The detector heat load is a relatively minor factor in the design, since the high efficiency of the dADR results in a heat output at $\sim 2.7 \mathrm{~K}$ of only about $0.2 \mathrm{~mW}$ (including parasitic heat loads and hysteretic heat generation). This represents only $3 \%$ of the unmargined capability of the iADR. At this early stage of mission design, it was deemed prudent to allocate $1 \mathrm{~mW}$ of the iADR's cooling power budget to heat rejection from the dADR.

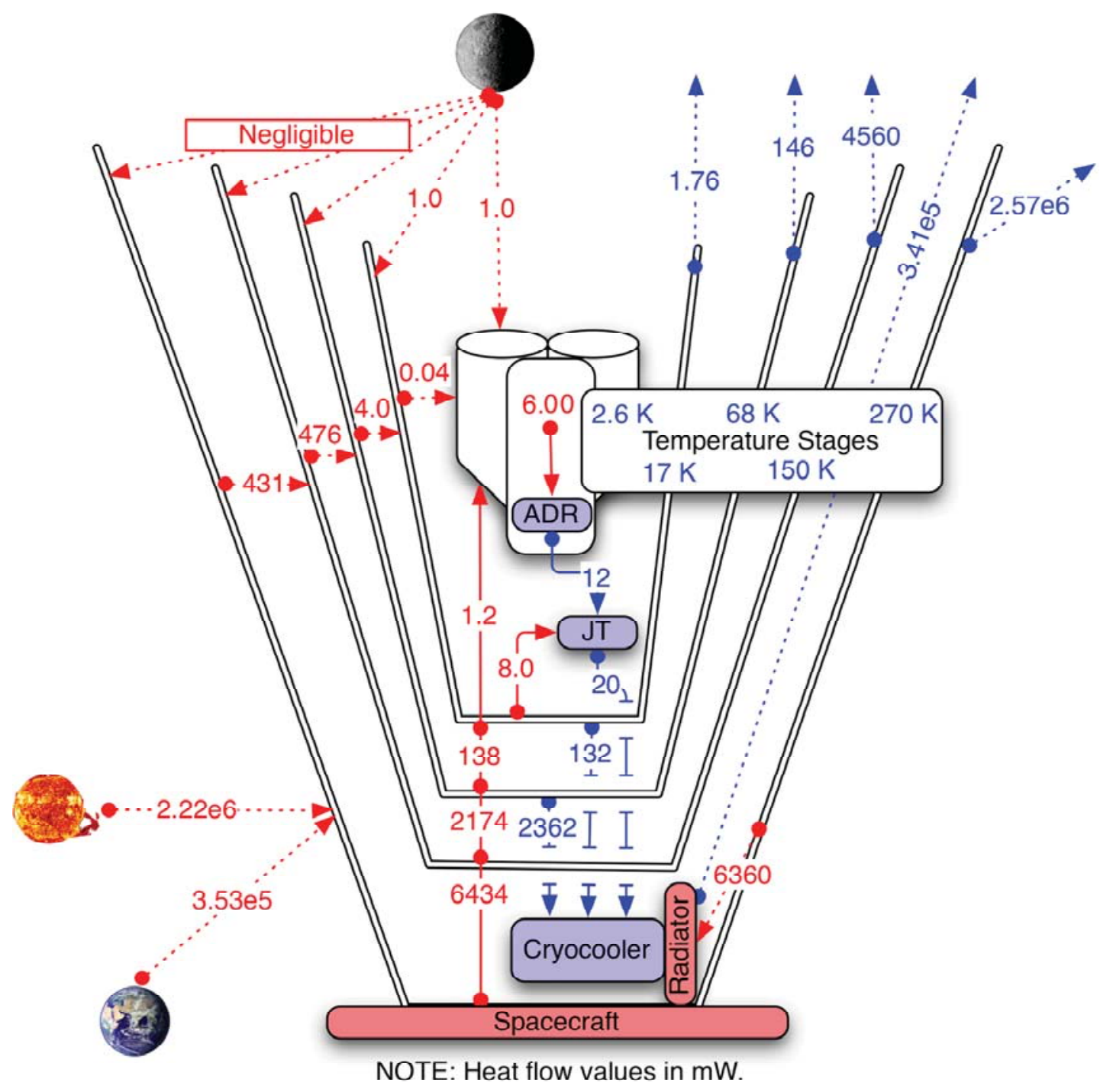

Figure 2. Heat Map of the PIXIE Cryogenic System. Red indicates heat inputs and blue shows heat outputs either radiated or absorbed by coolers. Dotted lines indicate radiative heat and solid lines indicate conducted heat flow. 
Table 1. Heat budgets for the dADR and iADR.

\begin{tabular}{|l|c|}
\hline \multicolumn{2}{|c|}{ dADR } \\
\hline Operating temperature & $0.100 \mathrm{~K}$ \\
\hline Duty cycle & $100 \%$ \\
\hline Temperature stability & $1 \mu \mathrm{K} \mathrm{rms}$ \\
\hline Detector heat load & $2 \mu \mathrm{W}$ \\
\hline Heat sink temperature & $2.66 \mathrm{~K}$ \\
\hline Heat load on heat sink & $<1.0 \mathrm{~mW}$ (time average) \\
\hline \multicolumn{2}{|c|}{ iADR } \\
\hline Operating temperature (see Section 2.4) & $2.66 \mathrm{~K}$ \\
\hline Duty cycle & $100 \%$ \\
\hline Heat load & $6.00 \mathrm{~mW}$ \\
\hline Input from dADR & $1.0 \mathrm{~mW}$ \\
\hline Conduction from 17 K & $1.2 \mathrm{~mW}$ \\
\hline Thermal control of optics & $2.85 \mathrm{~mW}$ \\
\hline Lunar irradiance (peak orbit average) & $1.0 \mathrm{~mW}$ \\
\hline Heat sink temperature & $4.5 \mathrm{~K}$ \\
\hline Peak heat load to sink & $12 \mathrm{~mW}$ \\
\hline
\end{tabular}

\section{1 iADR Configuration}

The driving requirement for the iADR is the peak heat reject rate of $12 \mathrm{~mW}$ [2]. This results from power constraints imposed by the PIXIE's spin (which reduces the time average output of the solar arrays) and the goal of operating during the eclipse season. According to mandatory design rules for this stage of mission development, the cryogenic system must be designed for $100 \%$ margin on all heat loads, therefore the iADR must actually be capable of $12 \mathrm{~mW}$ of heat lift at $2.66 \mathrm{~K}$, and the cryocooler capable of accepting a peak load of $24 \mathrm{~mW}$ from the iADR.

To see how this constrains the design, we note that a cooler operating between $2.66 \mathrm{~K}$ and $4.5 \mathrm{~K}$ with a cooling power of $6.0 \mathrm{~mW}$ at $100 \%$ (Carnot) efficiency (for the cold components only) would have a continuous heat reject rate of just over $10 \mathrm{~mW}$. This would seem to imply that the iADR must achieve an unrealistic efficiency of 85\%. However, this calculation is based doubly margined heat loads. The current best estimate for the heat output of the iADR is $0.2 \mathrm{~mW}$, and the dissipation needed for control of the optics is 1.85 $\mathrm{mW}$. Thus the unmargined cooling power requirement is $4.2 \mathrm{~mW}$ at $2.66 \mathrm{~K}$. Moreover, the optics dissipation can be reduced during periods of peak lunar input to further reduce the maximum heat load on the iADR. Consequently, the required efficiency of the iADR is less than $60 \%$, for which the entire cryogenic system still carries a margin of $100 \%$.

This has a significant impact on the architecture of the iADR. Continuous operation of an ADR can be achieved with 2 stages in either a series or parallel configuration. These are shown schematically in Figure 3. In the series configuration, one stage is held at constant 
temperature while the other shuttles back and forth in temperature, spending about half the time offloading heat at the cold end and half the time rejecting heat to the sink. At best, this can result in about $50 \%$ duty cycle for heat rejection, and a maximum efficiency of less than $50 \%$. In the parallel configuration, one stage absorbs heat from the load and controls its temperature while the other is rejecting heat, then the roles reverse. In this mode, the load is always being cooled, while heat is being rejected nearly continuously - realistically $\sim 80 \%$ of the time. The iADR can achieve $60 \%$ overall efficiency if each of the two stages can achieve $80 \%$ efficiency for heat absorption and rejection. At temperatures in the 2.6$4.5 \mathrm{~K}$ range, these kinds of efficiencies have already been achieved with laboratory ADR stages.

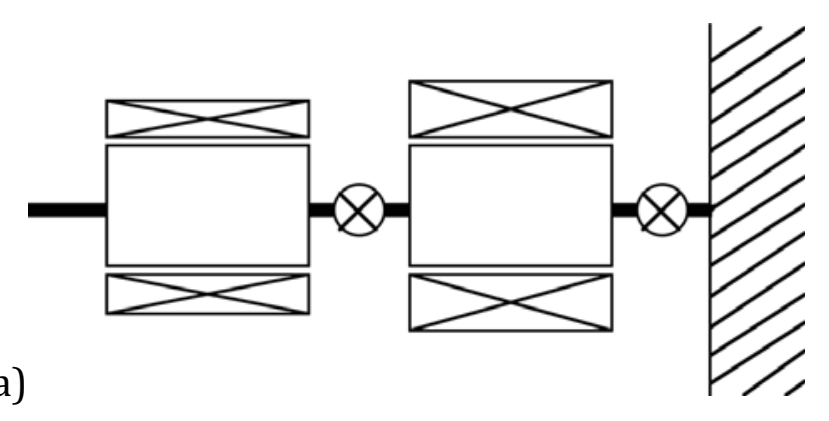

(b)

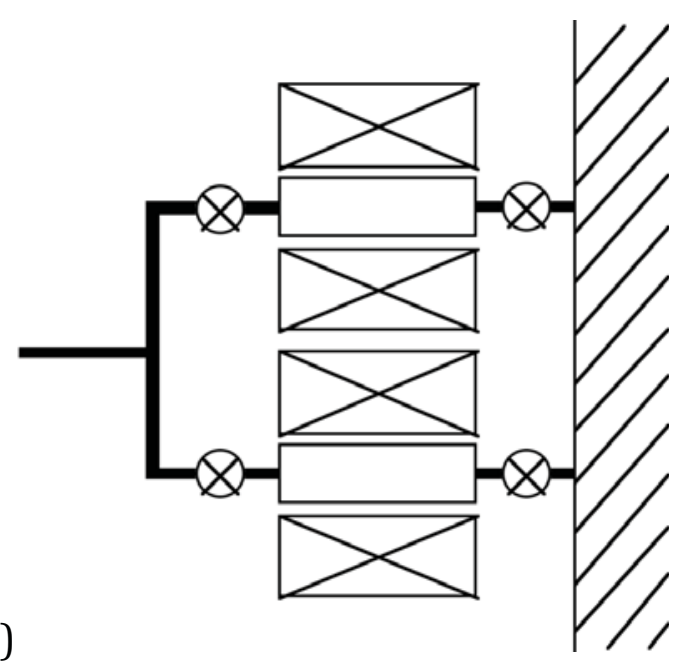

Figure 3. (a) Series and (b) parallel configurations for an ADR capable of continuous cooling.

The drawback to the parallel configuration is the need for 4 heat switches instead of 2 . The control system is also more complex as there is a handoff in temperature control each half cycle. However, there are benefits: the mass of each stage is half of what would be necessary in the series configuration, as are the heat fluxes. The latter contributes significantly to being able to meet the necessary efficiency for heat inflow/outflow.

For the refrigerant, gadolinium lithium fluoride (GLF)[3] has the highest entropy density of any material that has been used in space and/or laboratory ADRs in the temperature range from $2-5 \mathrm{~K}$. Its high density $\left(5.6 \mathrm{~g} / \mathrm{cm}^{3}\right)$ enables relatively high magnetic field $(3 \mathrm{~T})$ to be used, yet maintain low overall mass. The heat switches will all be gas-gap. The two switches connecting the salt pills to the heat sink will be passive[4], while those connecting the salt pills to the telescope thermal bus must be active.[5]

\section{2 dADR Configuration}


The cooling power requirement and presumed efficiency for the dADR are low compared to what has already been demonstrated in a developmental continuous ADR (CADR) [6] developed at NASA/Goddard Space Flight Center. This was a 4-stage assembly, using a linear thermal arrangement, that cooled to temperatures below $50 \mathrm{mK}$ using a $4.2 \mathrm{~K}$ heat sink. Using design rules that have been validated by the CADR, the PIXIE cooler requires only 3 stages to span $100 \mathrm{mK}$ to $2.66 \mathrm{~K}$. The lower cooling requirement means that the PIXIE stages can be scaled smaller, though less than proportional with cooling power required because the 3 coldest stages of the CADR operate over a wider temperature range. In practice, this just requires larger refrigerant mass and magnetic field for the 2 upper stages.

For stages 1 and 2, operating in the $100 \mathrm{mK}$ regime requires the use of a low density refrigerant like chrome potassium alum (CPA) or ferric ammonium alum (FAA). Entropy considerations favor FAA, but the long history within NASA/GSFC of using CPA for low temperature ADR stages and its compatibility with copper thermal buses provide a strong rationale for its use. As in the 4-stage CADR, stages 2 and 3 will use passive gas-gap heat switches, and to connect stage 1 to 2 , a superconducting heat switch using high-purity $\mathrm{Pb}$ wire will be used.

Design parameters for the dADR and iADR are given in Table 2 .

Table 2. Summary of design parameters for the dADR and iADR.

\begin{tabular}{|c|c|c|c|c|c|}
\hline Stage & Refrigerant & B (T) & Upper T (K) & Lower T (K) & Mass (kg) \\
\hline 1 & 20 g CPA & 0.1 & & 0.1 & 0.26 \\
\hline 2 & $20 \mathrm{~g} \mathrm{CPA}$ & 0.5 & 0.5 & 0.09 & 0.49 \\
\hline 3 & $30 \mathrm{~g} \mathrm{CPA}$ & 2 & 2.8 & 0.45 & 1.88 \\
\hline $4 \mathrm{a}$ & $100 \mathrm{~g} \mathrm{GLF}$ & 3 & 4.8 & 2.66 & 2.69 \\
\hline $4 \mathrm{~b}$ & $100 \mathrm{~g} \mathrm{GLF}$ & 3 & 4.6 & 2.66 & 2.69 \\
\hline Total & & & & & 8.00 \\
\hline
\end{tabular}

\subsection{ADR System Configuration}

While the PIXIE cooler uses 5 ADR stages, shown schematically in Figure 4, the continuous cooling produced by the iADR allows the two ADR assemblies to operate completely independently. Thus the "complexity" of the ADR and its control system is significantly reduced. Moreover, the two ADR systems can be independently developed and tested prior to integration with the telescope. 


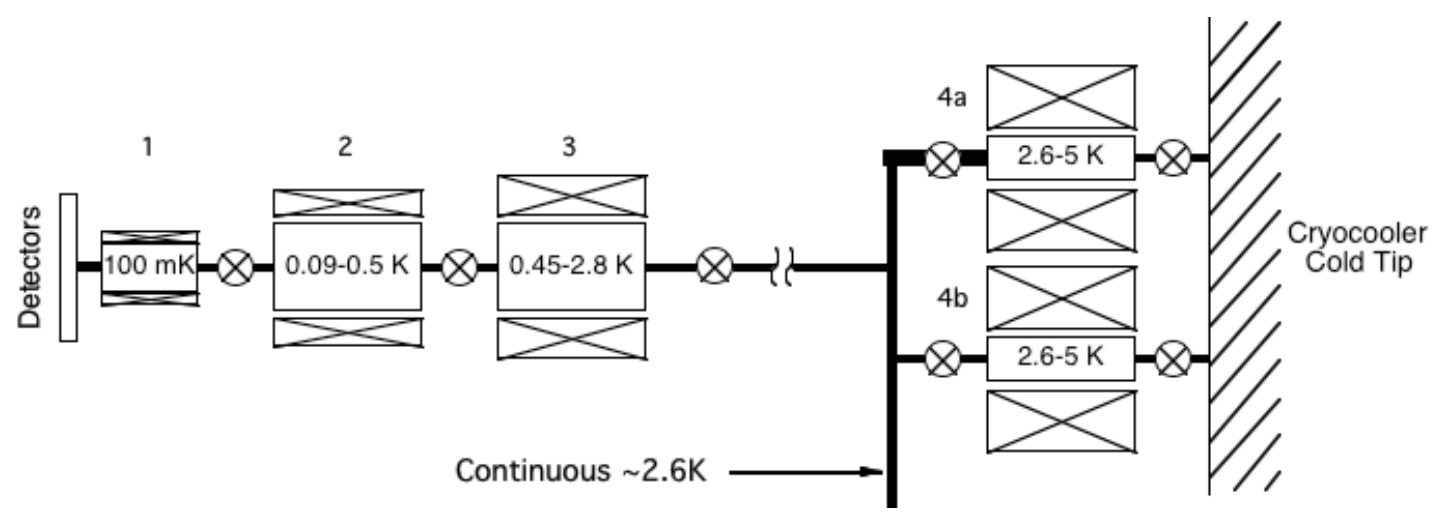

Figure 4. Schematic of the dADR (stages 1-3) and iADR (stages 4a-4b).

\subsection{Temperature Control of the Telescope and Optical Components}

The polarization signals from the CMB are extremely small. It is for this reason that the PIXIE telescope is cooled to the CMB temperature of $2.725 \mathrm{~K}$. Even so, gradients within the telescope and optics may contribute spurious signals. To separate these errors from the polarization signals, the telescope and major optical components (20 in all) will be dithered about the CMB temperature at unique frequencies. The typical excursion, $\Delta$, positive and negative, will be 5-10 $\mathrm{mK}$, at time scales of tens of minutes, but not commensurate with the orbit period.

For the optical components to be warmed above and cooled below the CMB temperature, the iADR must establish a low enough base temperature. Based on the final temperature selected, the thermal conductance of each component to the thermal bus will be tailored to yield a suitably short cooling time from $+\Delta$ to $-\Delta$. From the thermal conductance and the difference between the $\mathrm{CMB}$ and thermal bus temperatures, the average control heater input for each optical component can be calculated. More important than the total heater dissipation, $\dot{Q}$, which is simply the sum for all optical components, is entropy usage ( $\left.\dot{Q} / \mathrm{T}_{\text {bus }}\right)$ in the iADR to absorb this heat.

For thermal bus temperatures close to the CMB temperature, the thermal conductance of each optics link must be large in order to cool the component through a small temperature gradient. This yields large total heat flow at the average (CMB) temperature. As the thermal bus is lowered, total heat flow and entropy usage drop. Below an optimum temperature, where the heat flow stabilizes, the entropy usage will rise.

The optimal thermal bus temperature was determined to be $2.66 \mathrm{~K}$, for which the time average heater dissipation will be $1.85 \mathrm{~mW}$. The thermal budget for the iADR carries 2.85 $\mathrm{mW}$ for temperature control to provide margin against the inability to achieve very low thermal conductance for some very low mass components. Strictly speaking, this is not necessary since the $100 \%$ margin on ADR cooling power/heat rejection provides margin 
for such realities. However, the criticality of the temperature control scheme for removing instrument errors suggests the need for additional margin.

\section{0 iADR MODELING}

Operation of the iADR was modeled using control software and ADR simulators developed for the CADR R\&D program. The software has been validated through the design and build of several ADRs, including the CADR and Astro-H ADR. The model captures all aspects of the operation relevant to the thermodynamic performance, including internal thermal boundary resistance in the salt pills, heat switch transition times, and magnet/magnetic shield hysteresis.

Figure 5 shows the modeled operation of the iADR with a constant heat load of $5 \mathrm{~mW}$ (the total heat load without the additional margin on telescope/optics temperature control). Shown are the temperatures of stages $4 \mathrm{a}$ and $4 \mathrm{~b}$ through several cycles. The cycle period is 1600 seconds, during which each stage spends half the time absorbing heat from the thermal bus and half the time rejecting heat to the JT cooler. Each stage absorbs only a fraction of its total cooling capacity ( $\sim 5 \mathrm{~J}$ out of $7 \mathrm{~J}$ ) before recycling. This maintains some cooling reserve in the event of off-nominal (i.e. temporary) heat loads. It also allows the iADR to absorb larger heat loads during operation (i.e. the margined thermal control heat load).

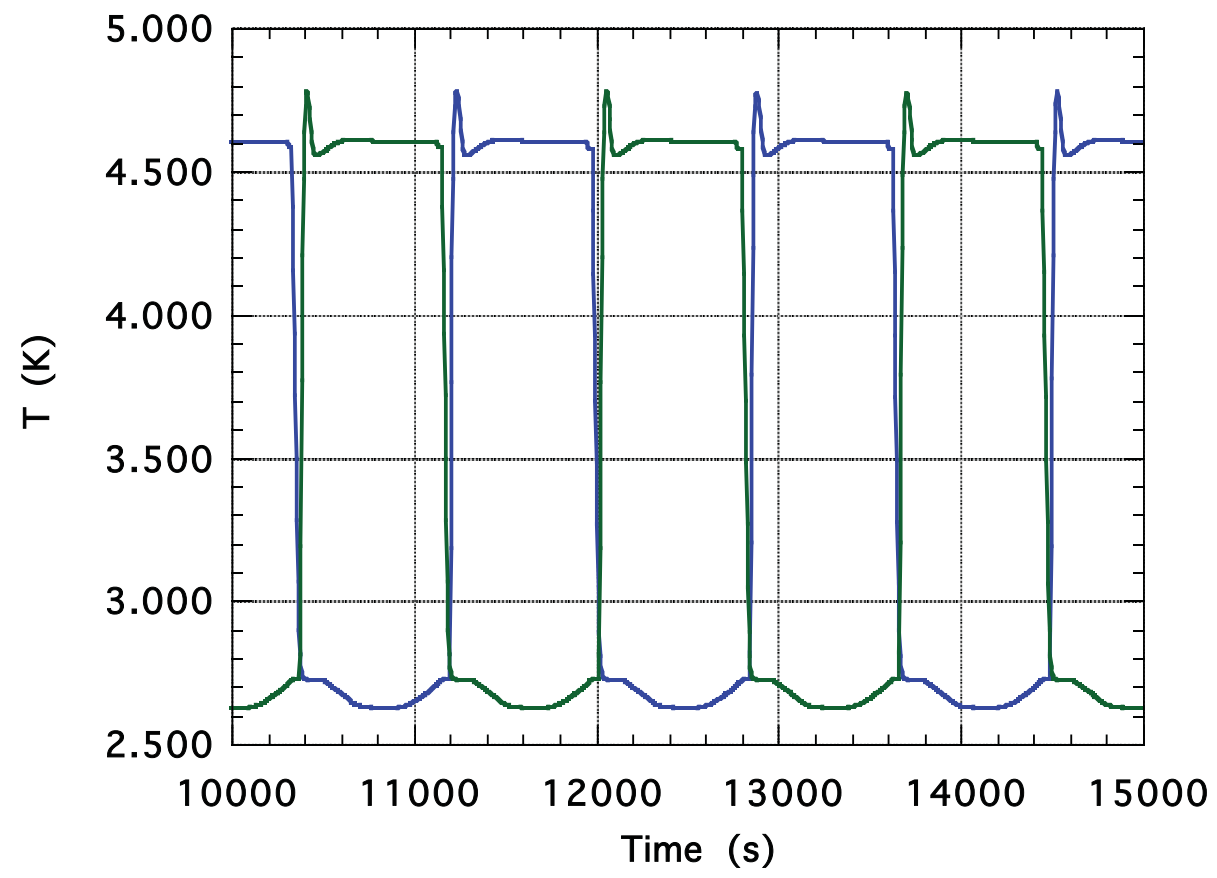

Figure 5. Modeled temperatures of the iADR stages through several cycles with a constant $5 \mathrm{~mW}$ heat load imposed. 
Figure 6 shows the instantaneous heat load to the JT cooler from the iADR. The time average is $9.8 \mathrm{~mW}$, while the peak momentarily exceeds $12 \mathrm{~mW}$. The difference between the two is due to the overhead operations of magnetizing each stage from $2.66 \mathrm{~K}$ to $4.5 \mathrm{~K}$, and subsequently demagnetizing over the same range. From the plot, it is obvious that the gap can be narrowed by fine-tuning the control parameters to achieve constant $12 \mathrm{~mW}$ heat rejection for the entire recycling operation. Less obvious is the ability to reduce the fraction of time taken up by overhead operation by extending the heat absorption and heat rejection phases. This can be accomplished by more deeply discharging each stage before it is recycled, or (worst case) adding refrigerant mass. Additional modeling is needed to test these cases.

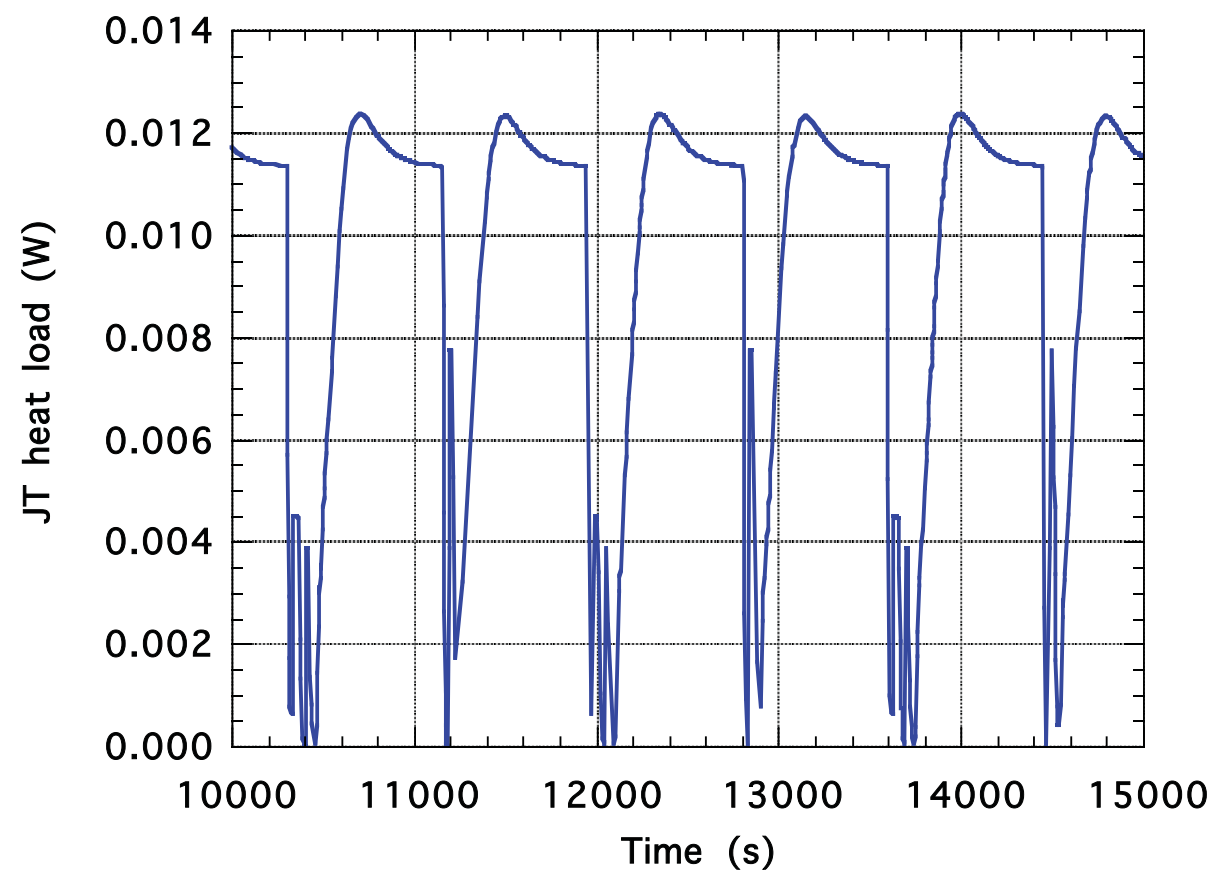

Figure 6. Modeled heat rejection rates of the iADR stages through several cycles with a constant $5 \mathrm{~mW}$ heat load imposed.

In summary, the model results show that the PIXIE ADRs can meet the current best estimate for instrument heat loads with $100 \%$ margin on cooling power and heat rejection, but at present cannot show $100 \%$ system-level margin with the additional margin on telescope/optics thermal control heater dissipation. It is expected that if PIXIE is selected from Phase A study under NASA's 2011 Mid-Ex AO, additional modeling and breadboard development will be done to demonstrate a system capable of meeting the more conservative requirements.

\subsection{SUMMARY}


The PIXIE mission presents a challenging set of cooling requirements: continuous cooling of detectors to $100 \mathrm{mK}$ and continuous cooling of its telescope and optics to near the CMB temperature of $2.725 \mathrm{~K}$. A low base temperature of $4.5 \mathrm{~K}$, from which the instrument cooler will operate, is provided by a set of nested thermal shields and a Stirling/JT cryocooler. Limited power resources require the instrument cooler to achieve very high efficiency, on the order of $60 \%$ of Carnot between $2.66 \mathrm{~K}$ and $4.5 \mathrm{~K}$.

The PIXIE instrument will use two ADR assemblies. Using two stages in a parallel configuration connected to the JT cooler, the iADR establishes a constant base temperature for the telescope and optics, and serves as a heat sink for the dADR. The dADR uses 3 stages in a linear configuration to operate continuously at $100 \mathrm{mK}$. The ADRs have been sized according to worst case, margined heat loads. Modeling shows that the PIXIE ADRs are close to meeting the heat lift requirements within the capability of the JT, after derating its cooling power by a factor of 2 .

During Phase A studies, additional modeling will be done to determine optimal control parameters for the ADRs, better estimates for heat loads, and the ability to phase various heat loads to reduce peak and time average values.

\subsection{REFERENCES}

[1] Kogut AJ, Chuss DT, Dotson JL, Fixsen DJ, Halpern M, Hinshaw GF, Meyer SM, Moseley SH, Seiffert MD, Spergel DN, and Wollack EJ, "The Primordial Inflation Explorer (PIXIE) Mission", Proc. SPIE 7731, 77311S (2010).

[2] DiPirro MJ, Fixsen D, Kogut A, LiX, Marquardt J, and Shirron PJ, "Design of the PIXIE Cryogenic System", submitted for publication in the proceedings of the 2011 Space Cryogenics Workshop, Coeur d'Alene, ID.

[3] Numazawa T, Kamiya K, Shirron PJ, DiPirro MJ, Matsumoto K, "Magnetocaloric Effect of Polycrystal $\mathrm{GdLiF}_{4}$ for Adiabatic Demagnetization Refrigeration", AIP Conference Proceedings, 850 (2006) 1579-1580.

[4] DiPirro, MJ, Shirron PJ, Tuttle JG, Canavan ER, "Design and Test of Passively Operated Heat Switches for 0.2 to 15 K", Adv. Cryo. Eng. 49 (2004) 436-442.

[5] Kimball MO and Shirron PJ, "Heat switches providing low activation power and quick switching time", submitted for publication in the proceedings of the $2011 \mathrm{CEC} / \mathrm{ICMC}$, Spokane, WA.

[6] Shirron, P.J., Canavan, E.R., DiPirro, M.J., Tuttle, J.G., and Yeager, C.J., “A Multi-Stage Continuous Duty Adiabatic Demagnetization Refrigerator," in Adv. Cryo. Eng. 45B, edited by Q.-S. Shu et al., Plenum, New York, 2000, pp. 1629-1638. 